Regular article

\title{
Quasi-optical analysis of a far-infrared spatio-spectral space interferometer concept
}

\section{Bracken ${ }^{\text {a,* }}$, C. O’Sullivan ${ }^{\text {a }}$, J.A. Murphy ${ }^{\mathrm{a}}$, A. Donohoe ${ }^{\mathrm{a}}$, G. Savini $^{\mathrm{b}}$, J. Lightfoot $^{\mathrm{c}}$, R. Juanola-Parramon ${ }^{\mathrm{b}}$, On behalf of the FISICA Consortium}

a Department of Experimental Physics, Maynooth University, Ireland

${ }^{\mathrm{b}}$ Department of Physics and Astronomy, University College London, United Kingdom

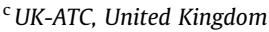

\section{A R T I C L E I N F O}

\section{Article history:}

Received 26 May 2016

Accepted 31 May 2016

Available online 3 June 2016

\section{Keywords:}

Far-infrared

FISICA

Interferometry

Double-Fourier

PyFInS (Python Far-Infrared Instrument

Simulator)

Optics

Quasi-optics

Physical optics

\begin{abstract}
A B S T R A C T
FISICA (Far-Infrared Space Interferometer Critical Assessment) was a three year study of a far-infrared spatio-spectral double-Fourier interferometer concept. One of the aims of the FISICA study was to setout a baseline optical design for such a system, and to use a model of the system to simulate realistic telescope beams for use with an end-to-end instrument simulator. This paper describes a two-telescope (and hub) baseline optical design that fulfils the requirements of the FISICA science case, while minimising the optical mass of the system. A number of different modelling techniques were required for the analysis: fast approximate simulation tools such as ray tracing and Gaussian beam methods were employed for initial analysis, with GRASP physical optics used for higher accuracy in the final analysis. Results are shown for the predicted far-field patterns of the telescope primary mirrors under illumination by smooth walled rectangular feed horns. Far-field patterns for both on-axis and off-axis detectors are presented and discussed.
\end{abstract}

(C) 2016 Elsevier B.V. All rights reserved.

\section{Introduction}

The FISICA project involved an international collaboration of researchers including leaders in the fields of far-infrared astronomy, cosmology, far-infrared instrumentation, optics, optical materials manufacture, and satellite positioning. FISICA aimed to identify the scientific questions related to high spatial resolution far-infrared observations, and to translate these questions into a technological definition of a far-infrared space-based mission, including a baseline telescope design. The work builds on previous far-infrared double-Fourier studies carried out by both European and US institutes, including the ESA Far-Infrared Interferometer (FIRI) Technology Reference Study (TRS) [1], the Space Infrared Interferometric Telescope (SPIRIT) study [2] (a candidate NASA Origins Probe mission), and the Balloon Experimental Twin Telescope for Infrared Interferometry (BETTII) [3]. For publications on the broader FISICA project see [4,5], for example.

\footnotetext{
* Corresponding author.

E-mail address: colm.bracken@nuim.ie (C. Bracken).

URL: https://www.maynoothuniversity.ie/experimental-physics/our-people/ colm-bracken (C. Bracken).
}

It has been long known that radiation in the far-infrared waveband can be used to probe many important astrophysical processes occurring in both the local and distant Universe. However, limitations due to diffraction mean that if resolutions $\left(\theta_{\min }=1.22 \lambda / D\right)$ of less than 1 arcsecond are to be reached in the far-infrared, then a primary mirror on the order of 50-100 m must either be built or synthesised. Atmospheric attenuation of far-infrared radiation, and the difficulties involved in using large mirrors in space leads to the requirement of space-based interferometry. Furthermore, if we are to at least match the spectral resolution of single dish far-infrared observatories such as Herschel [6,7], Spitzer [8] and SPICA [9], then a high-resolution spectroscopic technique must be used in combination with the spatial interferometer. The technique selected for the FISICA study was Fourier transform spectroscopy (FTS), and when spatial and spectral methods are used together the technique is called double-Fourier spatio-spectral interferometry [10].

One of the main drivers for a baseline optical layout for a farinfrared interferometer was the production of realistic aperture fields for use with the recently developed PyFIInS (Python Farinfrared Interferometer Instrument Simulator) software [11,12]. The PyFIInS simulator has thus far been capable of modelling the 
double-Fourier interferometry process, and reproducing sample sky maps while accounting for realistic sources of error and noise. To date though, the input primary mirror beams on the sky were those of uniformly illuminated apertures, and thus did not take account of optical aberrations, beam structure and polarisation effects of realistic detector beams, etc. Furthermore, it was clear that at such long wavelengths and propagation distances, diffraction would cause difficulties.

The main body of this paper starts with a description of the predetermined design parameters for the optical system of the interferometer resulting from the FISICA science case. A trade-off study between two very different interferometer design concepts is then presented, with one of the designs subsequently selected for further study. Following a discussion of results from approximate modelling methods such as ray tracing and Gaussian beams, an accurate PO (physical optics) model of the system is described. The PO model is ultimately used to propagate detector horn beams through the optical system, including the hub condensing optics, the variable interferometric baseline, and the light collecting telescopes. As such, the optical aberrations, beam truncation, and field structure that would be expected in a real system are accounted for in the predicted beam patterns shown in Fig. 6. Finally, a discussion of conclusions is given at the end of the paper.

\section{Light collecting telescopes}

\subsection{Broad optical design}

In the context of the FISICA study, the demands on sensitivity, resolution, and FoV (field of view) were well defined by the science case, and these requirements drove the initial optical design parameters. Two-metre primary mirrors (flux collectors) are required if integration times are to be kept within practical timescales. For example, for an interferometer with two mirrors $(d=2 \mathrm{~m})$, the average time needed per-pointing is $33 \mathrm{~h}$. This corresponds to approximately $4000 \mathrm{u}-\mathrm{v}$ sample points with two FTS scans per pair of $u-v$ points [13]. The most demanding science questions translate into the need to spatially resolve astrophysical objects of angular size $\approx 0.1-0.25$ arcseconds at wavelengths ranging from 25 to $200 \mu \mathrm{m}$, with a desire to extend the range to $400 \mu \mathrm{m}$. This sharp resolution at such long wavelengths requires interferometric baselines up to $B=100 \mathrm{~m}[13,4]$. The wide spectral coverage would likely be separated into three wavebands: (1) 25-50 $\mu \mathrm{m}$, (2) 50-100 $\mu \mathrm{m}$, (3) $100-200 \mu \mathrm{m}$, with a possible 4 th band of $200-400 \mu \mathrm{m}$, if feasible. Finally, science questions relating to mapping of the galactic centre call for a $1 \operatorname{arcmin}^{2}$ FoV. One on-axis single-mode coherent detector/horn assembly illuminating a $2 \mathrm{~m}$ primary mirror will yield a beam size on the sky on the order of a few arc seconds squared. However, filling the relatively large $\operatorname{arcmin}^{2}$ FoV with one such detector is not possible, and so a FPA (focal plane array) of single-mode or over-moded horns will be required. Thus, these values of $d=2 \mathrm{~m}, B_{\max }=100 \mathrm{~m}$, FoV $=\left( \pm 0.5^{\prime}\right)^{2}$, and the need for a FPA provided a clear starting point for the optical design.

Unlike imaging telescopes which focus a collimated beam onto a focal plane, the purpose of the light collecting telescopes in this instance is to convert a collimated beam into a smaller (de-magnified) collimated beam. This de-magnification is required in order to keep the size of the cooled hub optics small. Also, the beams must be propagated over distances up to $B_{1 / 2}=50 \mathrm{~m}$, before being combined in the hub craft. For each of the two beam paths a flat mirror oriented at $45^{\circ}$ to both the sky and the hub spacecraft is used to steer the beam toward the hub. De-magnification of the beams can be performed either before or after propagation over the semi-baseline. Fig. 1 (left) shows a design where demagnification is done before propagation over $B_{1 / 2}$. This was the option chosen by the FIRI study [15,1], where two on-axis afocal telescopes (pointed at the source) sample the $\mathrm{u}-\mathrm{v}$ plane and de-magnify the aperture fields. Fig. 1 (right) illustrates how de-magnification can alternatively be done after propagation over $B_{1 / 2}$, as selected by the balloon-borne BETTII mission [3] (due for launch in 2016). In this case two large flat siderostats (oriented at $45^{\circ}$ to the source) sample the $\mathrm{u}-\mathrm{v}$ plane and propagate the large beams toward the hub. Two afocal telescopes (off-axis layout for

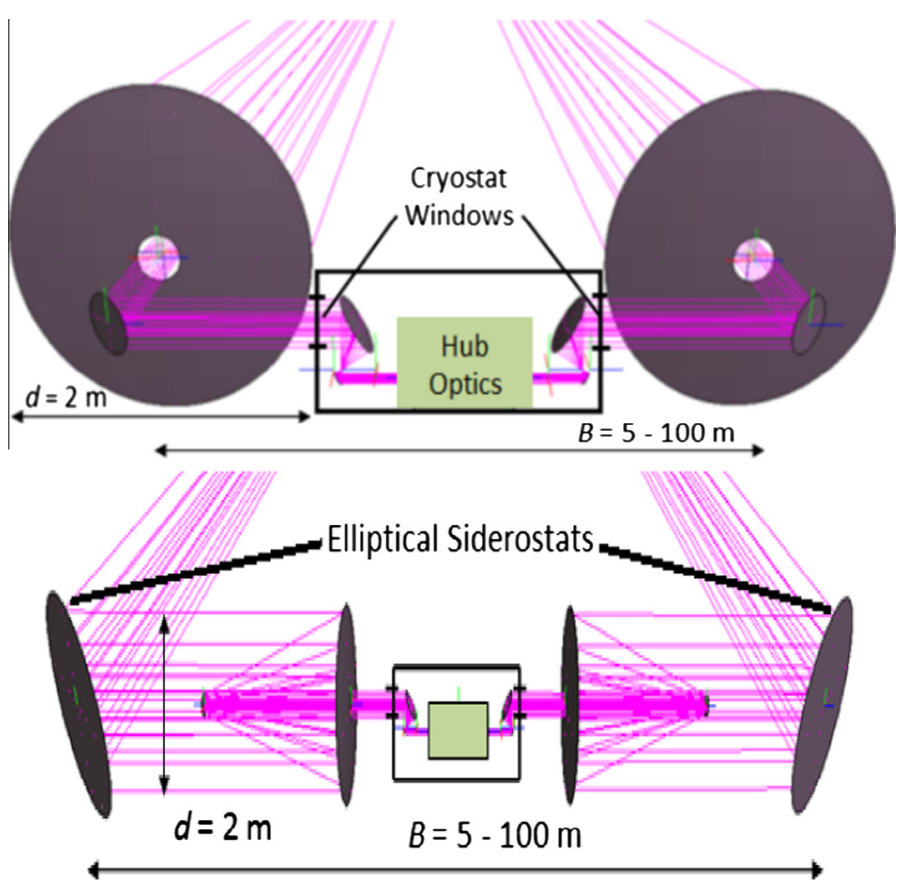

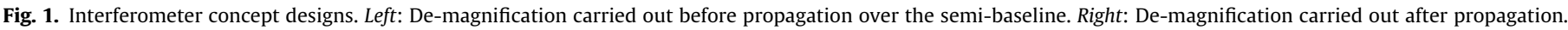
The required dimensions of the flat siderostats in the right figure are $a=2 \mathrm{~m}$ and $b=2.83 \mathrm{~m}$. (Pictures generated using GRASP software [14].) 

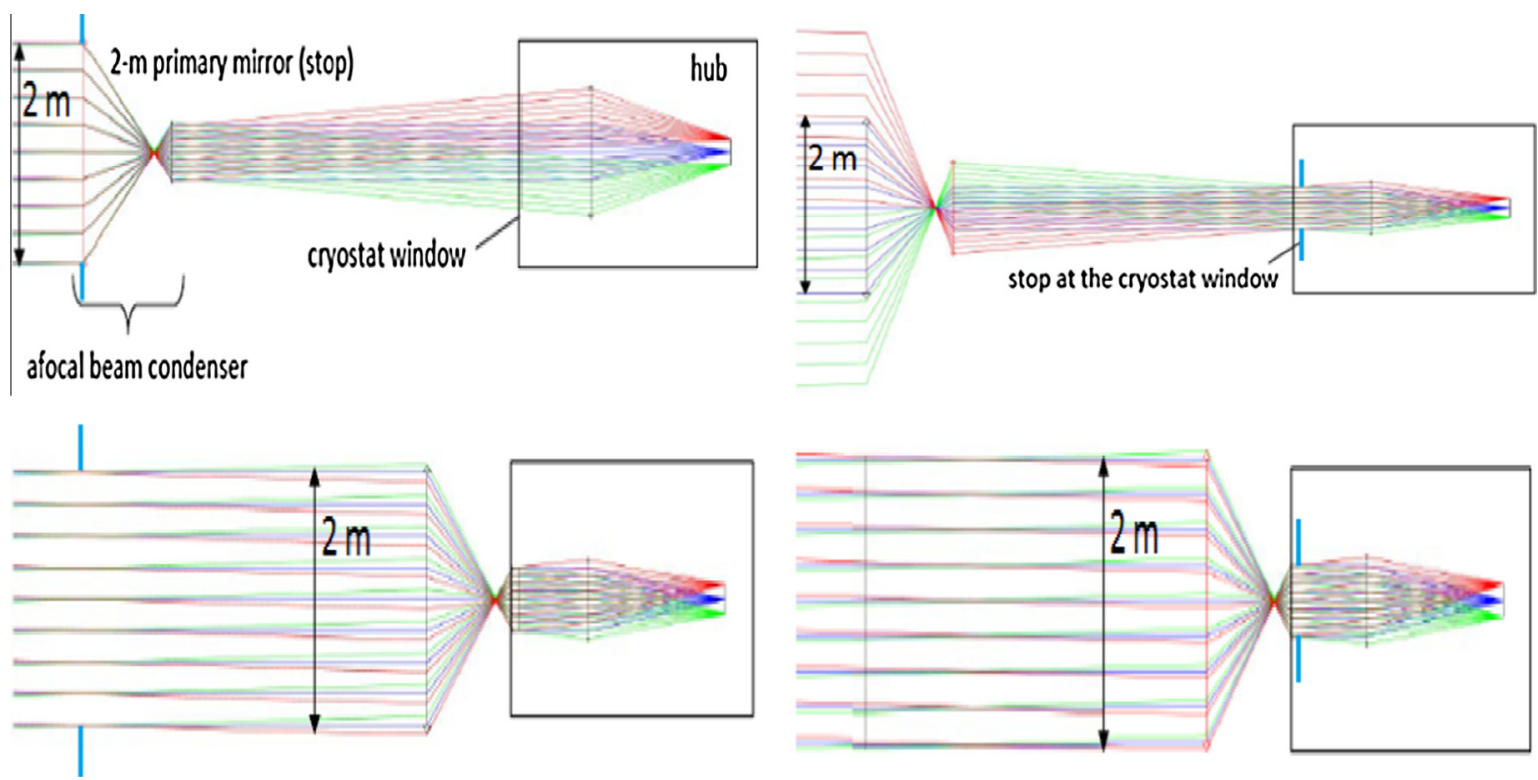

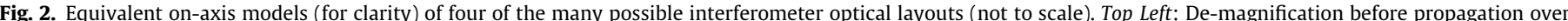

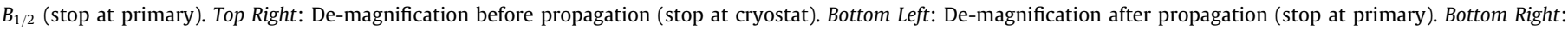
De-magnification after propagation (stop at cryostat).

BETTII) close to the hub then de-magnify the beams before they enter the hub.

\subsection{Trade-off analysis}

It was decided to compare optical designs where de-magnification is carried out at the primary flux collectors (which we will call type 1) with those where de-magnification is carried out close to the hub (we will call type 2). Using the ray tracing software Zemax, the predicted beam sizes at the hub cryostat window were compared for type 1 and type 2 designs. To be sure of comparing like-with-like, the analysis assumed the same parameters for the condensing optics in each case, namely de-magnification of $m=10$ and $F / 1.5$ primary.

These preliminary geometrical models show that there are significant differences for type 1 and type 2 systems, in terms of the minimum beam sizes at the hub. For type 1 designs, limiting the size of the primary mirrors to $2 \mathrm{~m}$ for a FoV of $\left( \pm 0.5^{\prime}\right)^{2}$ results in very large beam cross-sections at the hub window (over $400 \mathrm{~mm}$ ). This is simply due to a 'lever-arm' effect on the off-axis rays, where the propagation angles $\left(\phi_{f}\right)$ are magnified as $\phi_{f}=m \phi_{i}$, where $\phi_{i}$ are the angles of incidence (Fig. 2 (top left)). Given that the aperture of the hub cryostat must be cooled, its size should be kept as small as possible. If then, the cryostat window is to be the limiting factor, and the hub beam diameter is set to, say, $200 \mathrm{~mm}$, we find that the lever-arm effect demands the expansion of the primary mirrors to a diameter of at least $3.5 \mathrm{~m}$ (or even larger if there is to be no truncation of the longer wavelengths at the cryostat window) (Fig. 2 (top right)). There are of course other compromise options within (and beyond) the two extremes just described for a type 1 layout, and the exact design of a type 1 system will need to consider a trade-off between primary mirror size vs. cryostat window size.

In contrast to the type 1 design, if de-magnification is instead carried out close to the hub (type 2 ), a relatively small beam diameter at the hub cryostat window is achievable without increasing the size of the primary mirror. There is of course the addition of a large siderostat for each beam path, thus significantly increasing the mass of optical components required. As would be expected, due to the short distance between the telescope and hub for a type
2 design, there is little difference between placing the optical stop at the telescope primary mirror or at the hub window, unless a significantly smaller window is required. A summary of design conclusions is given in Table 1 .

A broader trade-off analysis of various afocal telescope types such as Gregorian vs. Cassegrain, and on-axis vs. off-axis (for a range of $\mathrm{F} / \# \mathrm{~s}$ ) was reported in a previous paper [16]. Ultimately, the final design for a particular mission will be chosen based on mass and compactness considerations vs. sensitivity and wavefront quality, etc. Given that FISICA was a study of a space-based system, and since mass limitations imposed by launch will likely exclude a type 2 design, it was a type 1 design that was selected for detailed modelling in this work. In order to predict the approximate size of the de-magnified FoV footprint at the hub (following propagation over $B_{1 / 2}$ ), a simple Gaussian beam model was used. This Gaussian beam model is approximate, in that it accounts for beam divergence, but neglects detailed beam-evolution effects due to diffraction.

The primary mirror of the type 1 system to be studied was set to $2 \mathrm{~m}$ (stop placed at primary), meaning that the cryostat window will need to be large. However its size could be reduced if a reduced FoV was acceptable (see Fig. 3 (right)). Again, these are details that would be refined for a real system design in the future.

\subsection{Optimising baseline telescope design}

As well as the magnification of the angle of off-axis rays by $m$ (described above), significant beam spreading due to diffraction can also occur at the longer wavelengths if the beams are condensed too much. An analysis was therefore carried out to determine the best value for $m$ from both a geometrical and diffractive point of view. The wavelength range considered was $20-200 \mu \mathrm{m}$ in steps of $20 \mu \mathrm{m}$, as well as a wavelength of $400 \mu \mathrm{m}$.

The on-axis beam from each of the telescopes can be approximated as a Gaussian [17] with beam radius $w$ that varies with propagation distance $B_{1 / 2}$, as shown in (1).

$w\left(B_{1 / 2}\right)=m \cdot w_{0} \sqrt{1+\left(\frac{\lambda B_{1 / 2}}{\pi w_{0}^{2}}\right)^{2}}=w_{0}^{*} \sqrt{1+\left(\frac{\lambda B_{1 / 2}}{\pi w_{0}^{2}}\right)^{2}}$ 
Table 1

Summary of the various optical formats for a two-telescope interferometer, and the implications.

\begin{tabular}{|c|c|c|}
\hline De-mag. at primary & Very large cryostat & $2 \mathrm{~m}$ primary (minimum \\
\hline Stop at primary & Window $(>400 \mathrm{~mm})$ & Mirror requirement) \\
\hline $\begin{array}{l}\text { De-mag. at primary } \\
\text { Stop at cryostat }\end{array}$ & $\begin{array}{l}\text { Quite large cryostat } \\
\text { Window }(200 \mathrm{~mm} \text { for } m=10)\end{array}$ & Large $3.5 \mathrm{~m}$ primary \\
\hline $\begin{array}{l}\text { De-mag. at hub } \\
\text { Stop at primary }\end{array}$ & $\begin{array}{l}\text { Moderate cryostat window } \\
\text { Requirement }(100 \mathrm{~mm})\end{array}$ & $\begin{array}{l}2 \mathrm{~m} \text { primary, plus large } \\
\text { Siderostat } a=2 \mathrm{~m}, b \approx 2.8 \mathrm{~m}\end{array}$ \\
\hline $\begin{array}{l}\text { De-mag. at hub } \\
\text { Stop at cryostat }\end{array}$ & $\begin{array}{l}\text { Smallest cryostat window } \\
\text { Requirement }(70 \mathrm{~mm})\end{array}$ & $\begin{array}{l}2.8 \mathrm{~m} \text { primary, plus large } \\
\text { Siderostat } a=2.8 \mathrm{~m}, b \approx 3.9 \mathrm{~m}\end{array}$ \\
\hline
\end{tabular}

where $\lambda$ is the wavelength of the light, $w_{0}$ is the radius of the best fit Gaussian to the uniformly illuminated primary mirror, and $w_{0}^{*}$ is the radius of the de-magnified Gaussian beam before propagation over the length of the variable semi-baseline $B_{1 / 2}$. If we then consider that the field intercepted by the primary mirror can be represented by a uniform disk of radius $a=1 \mathrm{~m}$, then the best fit Gaussian to the primary mirror has a beam radius of $w_{0}=0.892 \mathrm{~m}$. When the most off-axis fields (incident at angles $\phi= \pm 0.5^{\prime}$ ) are de-magnified by $m$, they too can be treated as simple Gaussian beams, but propagating at angles $m \phi$. The result is a Gaussian field distribution with a radial offset ( $\Delta w)$ from bore-sight, over an area (total footprint) of diameter $D$, as described by (2).

$$
\begin{aligned}
D\left(B_{1 / 2}\right) & =2 w\left(B_{1 / 2}\right)+2 \Delta w\left(B_{1 / 2}\right) \\
& =2 w_{0}^{*} \sqrt{1+\left(\frac{\lambda B_{1 / 2}}{\pi w_{0}^{2}}\right)^{2}}+2 B_{1 / 2} \sin (m \phi)
\end{aligned}
$$

Fig. 3 (left) shows how the FoV footprint size at the hub increases with propagation distance for a range of de-magnified beam width values. In this case $\lambda$ was set to $200 \mu \mathrm{m}$ since beam divergence will clearly be more severe at the longer wavelengths. As shown, the minimum footprint diameter that can be achieved at the hub for the maximum baseline is about $346 \mathrm{~mm}$, which corresponds to an initial de-magnified beam radius of about $89 \mathrm{~mm}$, or a de-magnification of $m=0.892 / 0.089=10$.

Fig. 3 (right) shows the minimum footprint diameter that can be achieved at the hub for increasing de-magnification and for a variety of wavelengths. In this case the propagation distance was set to the maximum semi-baseline $\left(B_{1 / 2}=50 \mathrm{~m}\right)$. Fig. 3 (right) also shows results for $\lambda=400 \mu \mathrm{m}$, where it is clear that extending the spectral coverage to this range will significantly increase the minimum beam size at the hub. On the other hand, if a smaller field of view is to be accepted (lowermost curves labelled $\lambda^{*}$ ), then a significantly smaller beam size can be achieved at the hub, even for wavelengths up to $400 \mu \mathrm{m}$. The smallest beam possible will clearly be for an on-axis field only, resulting in a beam radius at the hub (for $\lambda=200 \mu \mathrm{m}$ ) of $79.8 \mathrm{~mm}$ (not shown in Fig. 3). This optimised value corresponds to a beam at the telescope secondary mirror with a beam waist of $w_{0}=56.4 \mathrm{~mm}$, or de-magnification $m=892 / 56.4=15.8$. However, this scenario corresponds only to on-axis plane wave illumination, i.e. a single on-axis point source on the sky.

\section{Hub condensing optics}

\subsection{Hub propagation}

Based on the analysis described in Section 2.3, the minimum possible FoV footprint at the cryostat window will have a diameter of $346 \mathrm{~mm}$. Given that the footprint will have a Gaussian fall-off at its edge, an will therefore extend somewhat beyond $346 \mathrm{~mm}$, a cryostat window diameter of $400 \mathrm{~mm}$ was selected, with some level of truncation expected at the longest wavelengths and for the off-axis pixels. Following propagation across the variable baseline, and entry into the hub, the beams require further de-magnification so as to minimise the size of the cooled optical components within the hub. An off-axis Gregorian-like design with primary mirror diameter of $400 \mathrm{~mm}$ was chosen for the hub condensers, which relied on heritage from the FIRI study [15]. Table 2 shows the parameters for both the light collecting telescopes and the hub condensing optics.

Following de-magnification by the hub condensers, the beams, now approximately $30 \mathrm{~mm}$ in radius, are propagated through a series of optical elements including beam splitters, dichroics,
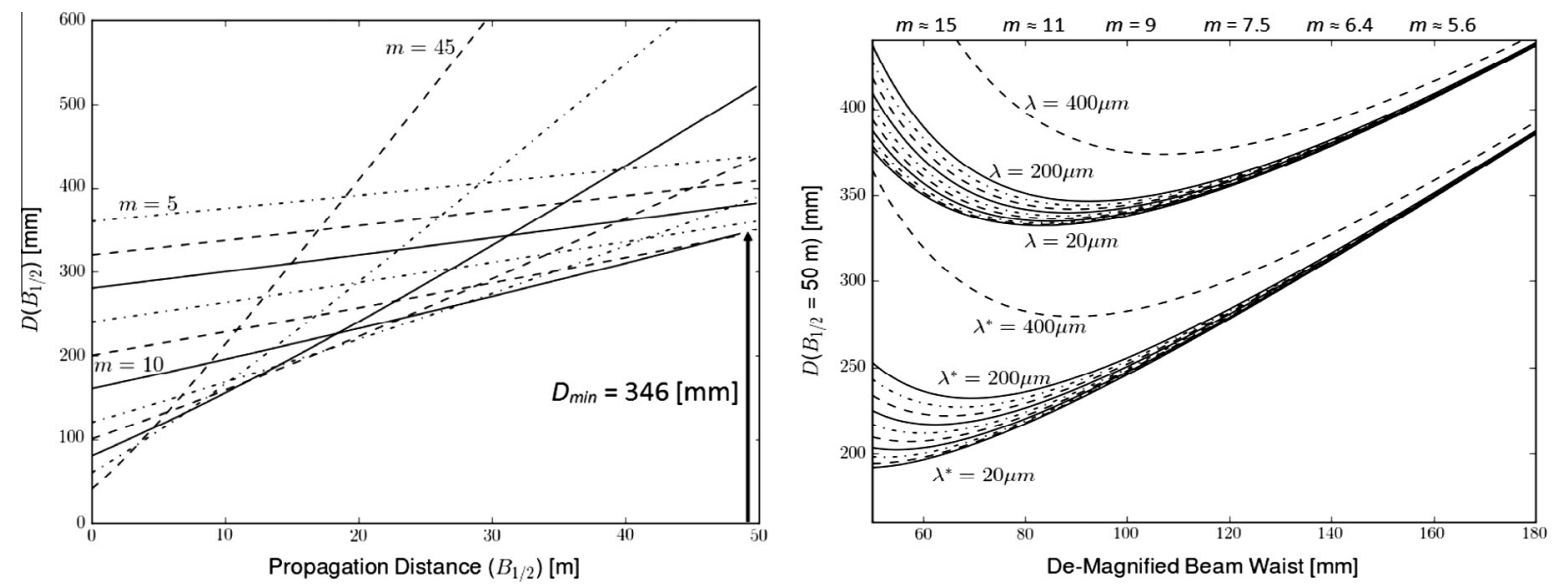

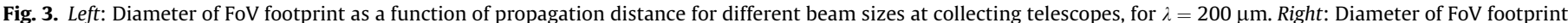

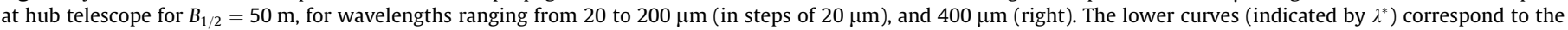
same wavelength values, but for a reduced FoV of $\pm 0.33^{\prime}$. 
Table 2

Parameters of both the light collecting telescopes and hub condensers.

\begin{tabular}{|c|c|c|c|c|c|}
\hline Primary telescopes (Cassegrain afocal) & Focal length (m) & De-magnification $\left(f_{1} / f_{2}\right)$ & Mirror radius (m) & $F / \#$ & Conic Const. \\
\hline Primary (M1) & 3 & 10 & 1 & 1.5 & -1 \\
\hline Secondary (M2) & 0.3 & & 0.1 & 1.5 & -1 \\
\hline Hub condensers (Gregorian afocal) & Focal length $(\mathrm{m})$ & De-magnification $\left(f_{3} / f_{4}\right)$ & Mirror radius $(\mathrm{m})$ & $F / \#$ & Conic const. \\
\hline Primary (M3) & 0.2 & 5 & 0.20 & 0.5 & -1 \\
\hline Secondary (M4) & 0.04 & & 0.043 & 0.465 & -1 \\
\hline
\end{tabular}

rooftop mirrors, and for one arm the FTS (Fourier transform spectrometer). Following combination of the two beams with a final beam combiner, the signal is focused onto the focal plane array by a final off-axis mirror. The mirror was given a focal length of $250 \mathrm{~mm}$ and angle of throw of $45^{\circ}$, and positioned $3 \mathrm{~m}$ from the hub window to allow for a realistic optical path length to facilitate all of the instrument specific optical elements. For the purposes of the current work, the final hub mirror was simply given an oversized radius of $600 \mathrm{~mm}$ to ensure a low level of truncation at all wavelengths. No attention is given here to the specifics of the other internal hub optics, since a number of optical layouts could be chosen for a range of science instruments based on heritage from the Herschel Space Observatory [18-20], or indeed the research to date on the planned SPICA mission $[21,22]$. Having determined a realistic baseline optical layout capable of satisfying the requirements of the FISICA study, attention was turned to the focal plane feed array in Section 3.2. Given the FoV required by the FISICA science case, and the resulting very large hub beams for wavelengths beyond about $200 \mu \mathrm{m}$, band 4 is ignored from this point as it will likely be unfeasible for a FISICA-type system.

\subsection{Focal plane fields and detector array}

Using the GRASP PO package [14], a total of nine linearly ploarised plane waves (representing the footprint of the FoV) were propagated through the system described in Table 2, from the primary flux collectors to the focal plane. The semi-baseline was set to an intermediary value of $25 \mathrm{~m}$. The aim of this was to analyse the focal plane fields corresponding to the FoV footprint, and then design an appropriate focal plane feed array for generating sky beams for input to the PyFInS simulator. A lens equivalent diagram (for clarity) is shown in Fig. 4 (left). The first plane wave represented an on-axis field, and the remaining eight corresponded to the limits of the FoV footprint in $\theta= \pm 0.5^{\prime}, \phi= \pm 0.5^{\prime}$, and $(\theta, \phi)=$ $\left( \pm 0.5^{\prime}, \pm 0.5^{\prime}\right)$. Fig. 4 (right) shows a mosaic of the 9 fields on the focal plane for $\lambda=200 \mu \mathrm{m}$, at which the beams would be expected to suffer the most significant level of diffraction and thus largest spot sizes. The footprint of a $3 \times 3$ array of rectangular horns is shown superimposed on the focal plane Airy patterns. Rectangular horns were chosen since they provide a higher packing density than conical horns. The horns were designed based on finding the best-fit Gaussian $(w=0.77 \mathrm{~mm})$ to the on-axis Airy pattern at the central wavelength of $150 \mu \mathrm{m}$, and then finding the horn that best couples to that Gaussian (Fig. 5 (right)). The rectangular horn that best couples to a Gaussian of radius $w$ has a height of $b=2 w$ and width of $a=\sqrt{2} b$ [23]. Thus, each of the horns in the $3 \times 3$ array was given an aperture of $a=2.18$ and $b=1.54 \mathrm{~mm}$. The filled band 2 FPA would consist of a $6 \times 6$ array of smaller feed horns, and band 1 would consist of a $12 \times 12$ array of even smaller horns.

\section{Detector to sky propagation}

\subsection{Detector feed overview}

In terms of simulating a detector beam for propagation through the interferometer system, no concern was given to the particular type of detector. A number of detector technologies would suit the FISICA sensitivity and bandwidth needs, with the leading technologies being TESs (transition edge sensors) and KIDs (kinetic inductance devices). TESs currently have a higher TRL (technology readiness level) [24], but KIDs also seem to provide promising technology due to their high suitability for arrays, and their high sensitivities [25]. For the purposes of the current work, each feed horn on the array was excited with a $T E_{10}$ transverse electric aperture field. Each horn aperture field was then propagated through the system shown in Table 2 and on to the sky. This analysis was also carried out using GRASP PO, with the aim of generating high accuracy realistic beams for use with the PyFIInS simulator.

\subsection{Beams on the sky}

Fig. 6 shows cuts through the far-field radiation patterns of a single telescope primary mirror illuminated by single feed horns, where the gain was calculated relative to an isotropic radiator. The results for the on-axis feed horn (Fig. 6 (top)) show no significant change in the on-axis intensity with changing semi-baseline distance, indicating no significant beam truncation. The relative
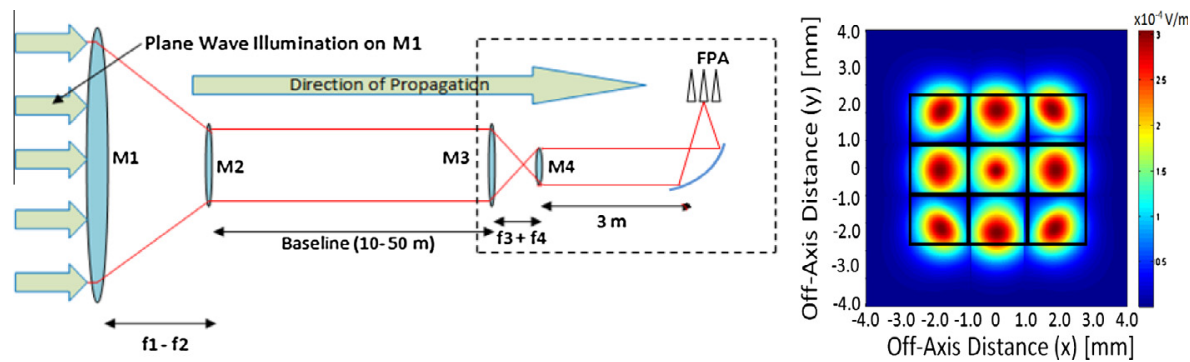

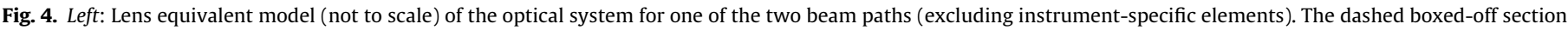
corresponds to the hub. Right: Focal plane fields for plane wave illumination of the $2 \mathrm{~m}$ primary, with footprint of feed horn array superimposed. 

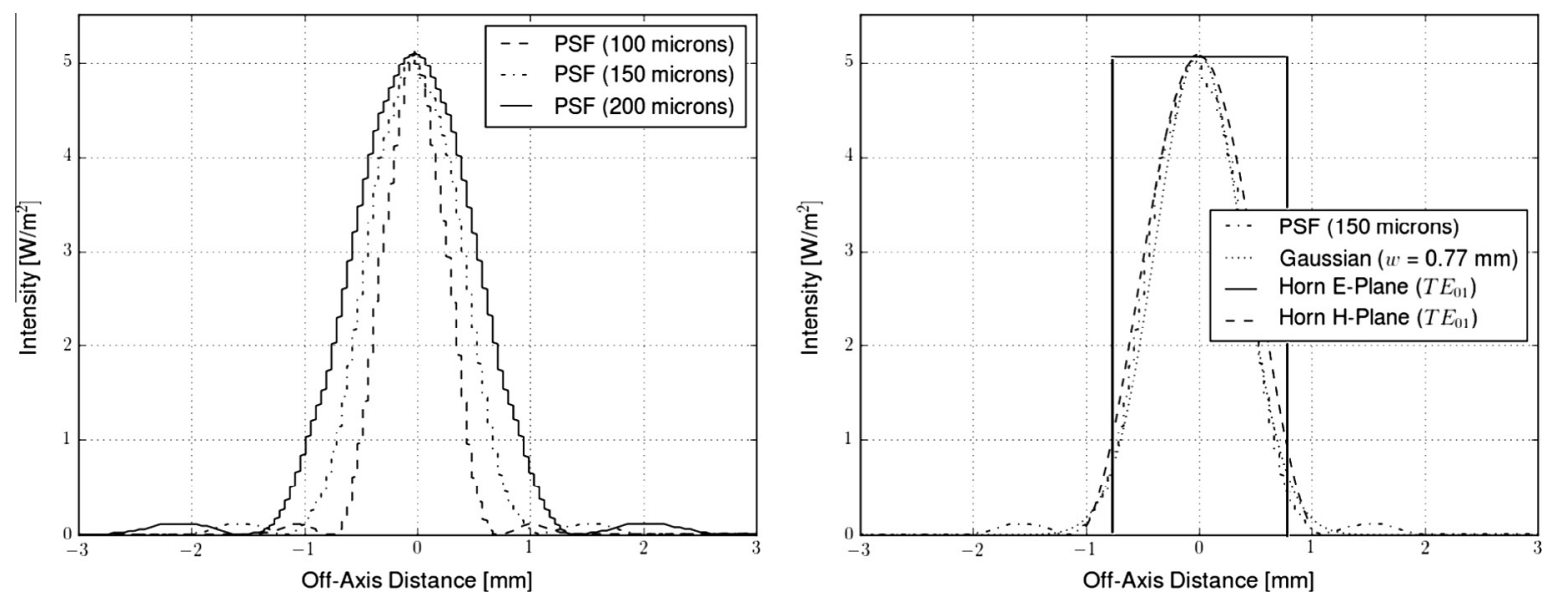

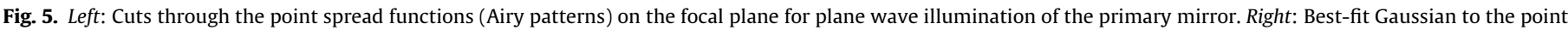
spread function for the central wavelength of band $3(\lambda=150 \mu \mathrm{m})$, and rectangular horn field cuts through $E$ and $H$ planes.
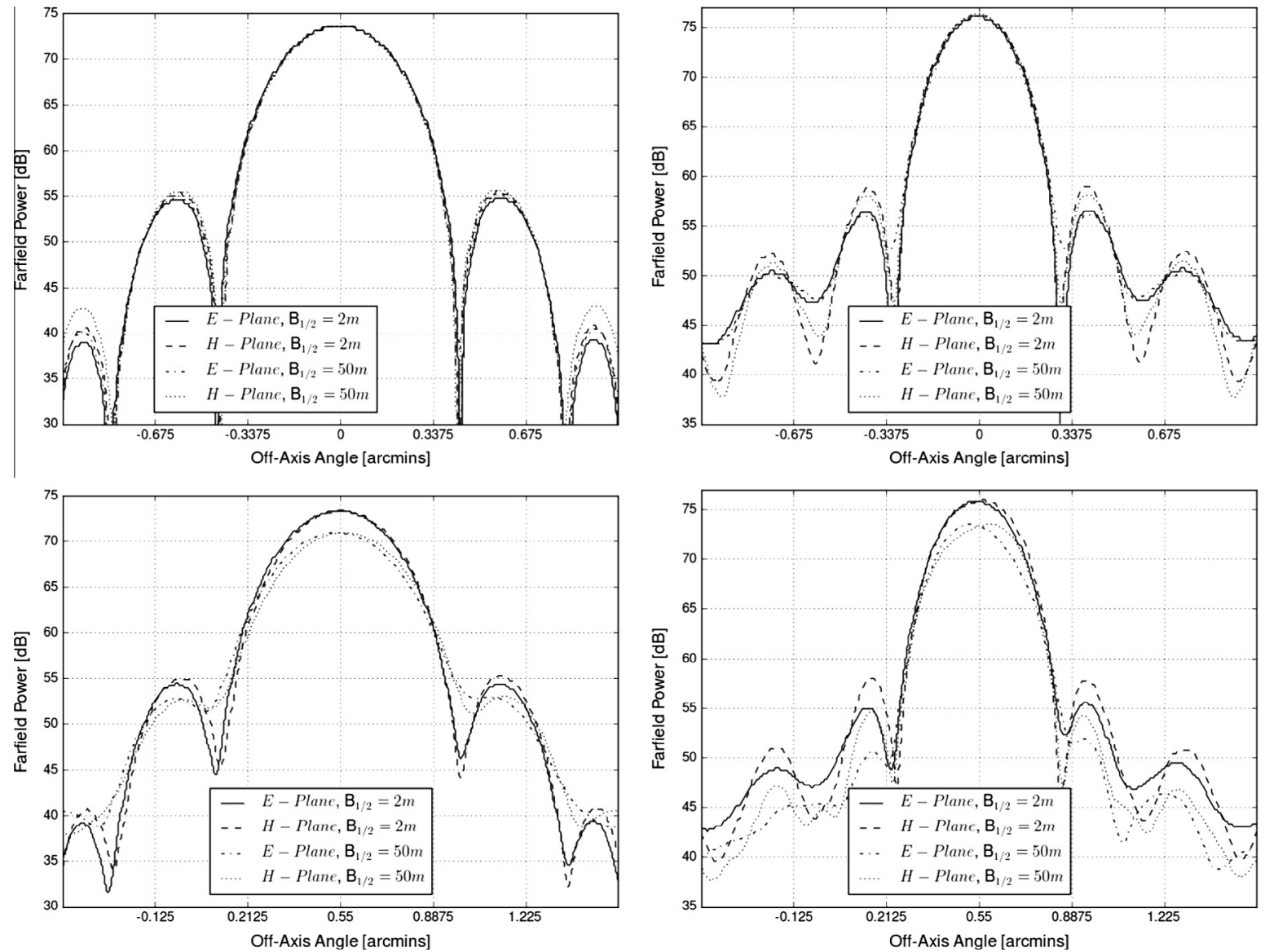

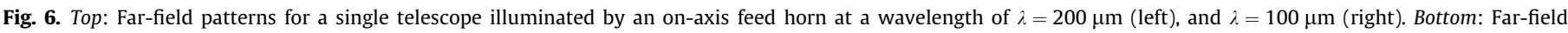
patterns for a single telescope illuminated by the furthest off-axis feed horn at a wavelength of $\lambda=200 \mu \mathrm{m}$ (left), and $\lambda=100 \mu \mathrm{m}$ (right).

increase in on-axis gain at the shorter wavelength (Fig. 6 (top right)) is to be expected, as would be the case for a conventional radiating single mirror/dish.

The results for the furthest off-axis pixel (Fig. 6 (bottom)) show a decrease (about $2 \mathrm{~dB}$ ) in gain for $B_{1 / 2}=50 \mathrm{~m}$ compared to $B_{1 / 2}=2 \mathrm{~m}$, which indicates some level of beam truncation at longer baselines. In fact the relative decrease is roughly the same for $\lambda=200 \mu \mathrm{m}$ and $\lambda=100 \mu \mathrm{m}$, indicating the truncation is primarily a result of the off-axis lever-arm effect, rather than diffraction which would be wavelength dependent. Fig. 7 again shows the single telescope far-field patterns, but for varying hub mirror diameter. If $>99 \%$ coupling at all wavelengths and for all pixels is to be achieved, a hub mirror with a diameter of $520 \mathrm{~mm}$ will ultimately be required. Due to the beam patterns significantly changing in structure with varying wavelength, separate beam data were generated for the shortest, central, and longest wavelength of band $3(\lambda=100,150$, and $200 \mu \mathrm{m})$ for input to the PyFInS instrument simulator. Based on these sample beams, the 

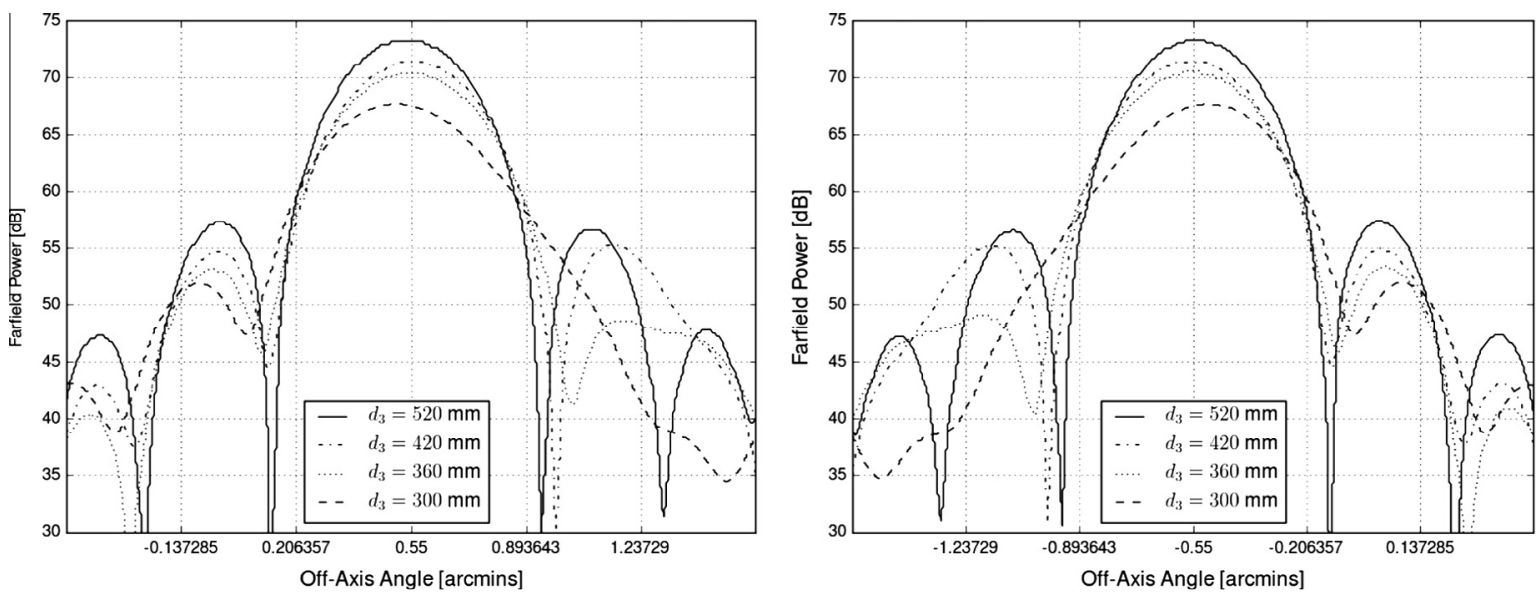

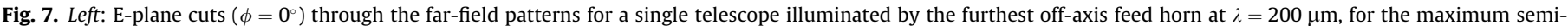
baseline $\left(B_{1 / 2}=50 \mathrm{~m}\right)$. The diameter $\left(d_{3}\right)$ of the hub primary mirror $\left(M_{3}\right)$ was varied. Right: H-plane cuts $\left(\phi=90^{\circ}\right)$.
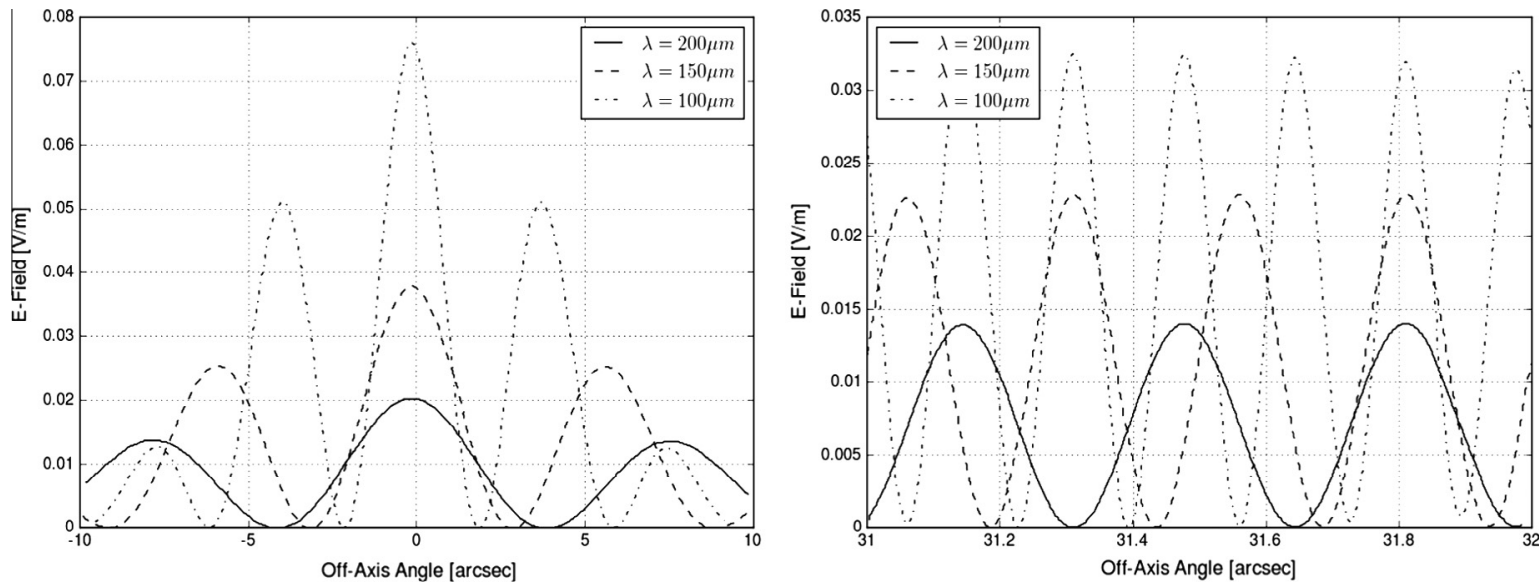

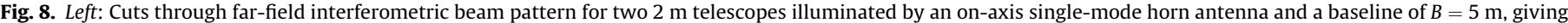

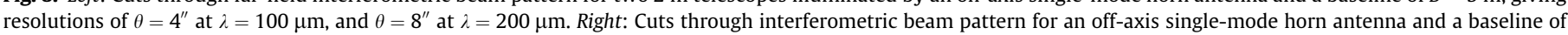
$B=50 \mathrm{~m}$, giving resolutions of $\theta=0.4^{\prime \prime}$ at $100 \mu \mathrm{m}$, and $\theta=0.8^{\prime \prime}$ at $200 \mu \mathrm{m}$, as would be expected.

simulator then interpolates beam patterns for a larger number of wavelength samples.

\subsection{Fringe visibility}

The predicted far-field interferometric beam patterns can also be calculated from the PO models, where the horn aperture fields are split into two beams, and each beam is then propagated through one of the two beam paths. By comparing the central peak to the first trough, we can roughly calculate fringe (or interferometric) visibility for each of the pixels at a given wavelength using (3).

$V=\frac{I_{\max }-I_{\min }}{I_{\max }+I_{\min }}$

However, the precise value of fringe visibility for a spatial interferometer is baseline dependent, and calculating it requires a more in-depth analysis and a metric called the mutual coherence function [26]. As can be seen in Fig. 8 (left) for an on-axis pixel and a relatively small baseline of $5 \mathrm{~m}$, the visibility function falls off due to the beam envelope corresponding to the beam pattern of a single $2 \mathrm{~m}$ mirror. This is also the case for larger baselines, although it is difficult to see in Fig. 8 (right) as the plot only extends over the central portion of the beam envelope. Furthermore, while the first troughs about the central peak do have minima of roughly zero, the troughs at larger angular separation do not quite reach zero. Any non-ideal behaviour in the interferometer is expected to cause a reduction in the visibility, in particular causing the nulls in the fringes to be partially filled in. This is due to non-destructive interference between the two non-identical beams on the sky.

Rather than struggling with precise definitions of fringe visibility, we can instead generate realistic beam data similar to those shown in Fig. 6, and allow PyFInS to simulate the doubleFourier process for observation of a particular sky scene. The details of the re-constructed sky maps and spectra can then inform on the level of visibility.

\section{Conclusions}

As part of the three year FP7 FISICA study, a design for a long baseline $(B=5-100 \mathrm{~m})$ far-infrared spatio-spectral doubleFourier interferometer concept was put forth. The FISICA science case called for a wavelength range of $\lambda=25-200 \mu \mathrm{m}$, likely split into three bands of 25-50, 50-100, and 100-200 $\mu \mathrm{m}$. An extended wavelength range up to $\lambda=400 \mu \mathrm{m}$ was also desirable for the science, but was deemed infeasible due to level of diffraction and resulting large hub beams. An interferometric baseline range up to $100 \mathrm{~m}$, and a FoV of $\left( \pm 0.5^{\prime}\right)^{2}$ were stated in the FISICA science 
case, and sensitivity requirements called for primary mirror diameters of $2 \mathrm{~m}$. This paper detailed how this two-telescope (and central hub) design was analysed using a number of computational methods. A fast ray tracing technique was used to show that if the telescope primary mirror diameters are set to $2 \mathrm{~m}$, then the size of the hub aperture must be very large (at least $400 \mathrm{~mm}$ ). An approximate Gaussian beam model which accounts for beam divergence was then used to quickly optimise the demagnification power $m$ of the light collecting telescopes. For the required FISICA FoV, a value of $m=10$ was found to provide the smallest possible beam sizes at the hub aperture, yielding a FoV footprint with a Gaussian diameter of $346 \mathrm{~mm}$ for a wavelength of $\lambda=200 \mu \mathrm{m}$ and a semi-baseline of $B_{1 / 2}=50 \mathrm{~m}$. It was shown that a significantly smaller hub aperture would be possible (even for $\lambda$ up to $400 \mu \mathrm{m}$ ) if a smaller FoV was acceptable.

Based on the ray tracing and Gaussian beam analysis, a baseline design was decided upon. Two on-axis Cassegrain afocal light collecting telescopes $(d=2 \mathrm{~m})$, with a de-magnification of $m=10$ and relatively standard F/1.5 are combined with a flat $45^{\circ}$ mirror to steer the beams toward the hub. In order to achieve small beams for propagation through the hub optics, the hub beams are further de-magnified by $m=5$ using two off-axis Gregorian afocal telescopes. A final off-axis parabolic mirror is then used to focus the fields onto a $3 \times 3$ array of rectangular feed horns. The horns were given apertures of $a=2.18 \mathrm{~mm}$ and $b=1.54 \mathrm{~mm}$ to maximise coupling with the on-axis Airy pattern at a wavelength of 150 $\mu \mathrm{m}$. A propagation distance of $3 \mathrm{~m}$ between the hub condensing optics and the final parabolic mirror was defined, to account for the optical path length of the hub optics.

Due to the long wavelengths involved, and thus the possible inaccuracies inherent in the approximate analysis techniques described, an accurate model of the optical system was built using the GRASP PO package. The PO model was used to generate realistic primary mirror far-field patterns for use with PyFIIns, an endto-end far-infrared interferometer instrument simulator. The design parameters shown in Table 2 result in a system capable of producing good predicted beam patterns on the sky for on-axis pixels at both ends of band $3(\lambda=100-200 \mu \mathrm{m})$, and for the large baselines demanded by the resolution requirements of FISICA. However, there is some level of beam truncation for the off-axis pixels at all wavelengths, which results in a loss in gain of about $2 \mathrm{~dB}$ for the most off-axis pixels of the array. Throughput can be increased to above $99 \%$ for all pixels by increasing the diameter of the hub window to about $520 \mathrm{~mm}$, although the size of the hub optics may well be the limiting factor in a real system. As such, a future physical system will likely require some alterations to the optical design described in this paper, and will further rely on a trade-off between mass and volume limitations, and the allowable levels of wavefront error at the shortest wavelengths and beam truncation at the longest wavelengths.

\section{Acknowledgements}

This research has received funding from the European Union's Seventh Framework Programme (FP7/2007-2013) under FISICA grant agreement No. 312818. The corresponding author also acknowledges the Irish Research Council for providing additional funding for this work.

\section{References}

[1] F. Helmich, R. Ivison, FIRI - a far-infrared interferometer, Exp. Astron. 23 (1) (2009) 245-276, http://dx.doi.org/10.1007/s10686-008-9100-2.
[2] D. Leisawitz et al., The space infrared interferometric telescope (SPIRIT): highresolution imaging and spectroscopy in the far-infrared, J. Adv. Space Res. 40 (1) (2007) 689-703, http://dx.doi.org/10.1016/j.asr.2007.05.081.

[3] S. Rinehart et al., The balloon experimental twin telescope for infrared interferometry (BETTII): an experiment for high angular resolution in the farinfrared, Publ. Astron. Soc. Pacific 126 (941) (2014) 660-673, http://dx.doi.org/ 10.1086/677402.

[4] G. Savini, P. Ade, N. Baccichet, C. Bracken, et al., Progress in the critical assessment for a far-infrared space interferometer with double fourier modulation (FP7-FISICA), in: OSA Technical Digest, Optical Society of America Imaging FTS in Astronomy (FT4A), 2015, pp. FT4A.3, http://dx.doi. org/10.1364/FTS.2015.FT4A.3.

[5] V. Iafolla, E. Fiorenza, L. Iafolla, C. Lefevre, C. Magnafico, F. Santoli, L. Spinoglio, FISICA (far infrared space interferometer critical assessment) metrological problems and system requirements for interferometric observations from space, in: Proceedings of IEEE Metrology for Aerospace (MetroAeroSpace) 2014, pp. 161-166, http://dx.doi.org/10.1109/MetroAeroSpace.2014.6865913.

[6] M. Servillat, A. Coleiro, S. Chaty, F. Rahoui, J. Zurita-Heras, Herschel observations of dust around the high-mass X-ray binary GX 301-2, Astrophys. J. 797 (2) (2014) 114, 10 pp, http://dx.doi.org/10.1088/0004$637 X / 797 / 2 / 114$

[7] I. Oteo, Dust correction factors over $0<z<3$ in massive star-forming galaxies derived from a stacking analysis of Herschel data, Astron. Astrophys. 572 (2014) L4, 12 pp, http://dx.doi.org/10.1051/0004-6361/201424607.

[8] M. Werner, G. Fazio, G. Rieke, T. Roellig, D. Watson, First fruits of the Spitzer space telescope: galactic and solar system studies, Ann. Rev. Astron. Astrophys. 44 (1) (2006) 269-321.

[9] J. Goicoechea, P.R.K. Isaak, L. Spinoglio, B. Swinyard, The SPICA Assessment Study Report, Prepared by the ESA SPICA Study Team, The SPICA Telescope Science Study Team, The JAXA/ISAS SPICA Team and the SAFARI Consortium.

[10] N. Elias-II, N. Harwit, D. Leisawitz, S. Rinehart, The mathematics of doubleFourier interferometers, Astrophys. J. 657 (2) (2007) 1178-1200, http://dx.doi. org/10.1086/510878.

[11] R. Juanola-Parramon, A Far-infrared Spectro-spatial Space Interferometer. Instrument Simulator and Testbed Implementation, Ph.D. Thesis, UCL (University College London), 2014.

[12] J. Lightfoot, R. Juanola-Parramon, G. Savini, FIInS - a python simulator for a farinfrared double fourier interferometer in space, in: OSA Technical Digest, Optical Society of America Hyperspectral Imaging and Sounding of the Environment (JM3A), 2015, pp. JM3A.9, http://dx.doi.org/10.1364/FTS.2015. JM3A.9.

[13] L. Spinoglio, D. Schito, S. Pezzuto, W. Holland, on behalf of the FP7-FISICA Consortium, D1.1: Definition/update of Key Science Questions and Relevant Data Products, FP7-FISICA Consortium. <http://www.homepages.ucl.ac.uk/ ucapgsa/Test_docs/D1.1_submitted.pdf>.

[14] K. Pontoppidan, GRASP Technical Description, TICRA.

[15] E.C.D. Facility, FIRI - Far Infrared Interferometer, CDF Study Report CDF-49(A), 2006, pp. 130-131.

[16] C. Bracken, on behalf of the FP7-FISICA Consortium, Optical and quasi-optical analysis of system components for a far-infrared space interferometer, in: Proceedings of SPIE 9362 Terahertz, RF, Millimeter, and Submillimeter-Wave Technology and Applications VIII (93620N), 2015, 12 pp, http://dx.doi.org/10. $1117 / 12.2076385$.

[17] P. Goldsmith, Quasi-optical techniques, Proc. IEEE 80 (11) (1992) 1729-1747, http://dx.doi.org/10.1109/5.175252.

[18] A. Poglitsch et al., The photodetector array camera and spectrometer (PACS) on the Herschel space observatory, Astron. Astrophys. 518 (L2) (2010) L2, 12 pp, http://dx.doi.org/10.1051/0004-6361/201014535.

[19] M.J. Griffin et al., The Herschel-SPIRE instrument and its in-flight performance, Astron. Astrophys. 518 (L3) (2010) L3, 7 pp, http://dx.doi.org/10.1051/0004$6361 / 201014519$.

[20] N. Trappe et al., Quasi-optical analysis of the HIFI instrument for the Herschel Space Observatory, in: Proceedings of SPIE 4876 Optics and Photonics Technologies and Applications, pp. 1091, http://dx.doi.org/10.1117/12. 463910.

[21] W. Jellema et al., The optical design concept of SPICA-SAFARI, in: Proceedings of SPIE 8442 Space Telescopes and Instrumentation 2012: Optical, Infrared, and Millimeter Wave, 2012. 84420SI, 9 pp. http://dx.doi.org/10.1117/12. 927153.

[22] H. Matsuhara et al., Cooled scientific instrument assembly onboard SPICA, in: Proceedings of SPIE 8442 Space Telescopes and Instrumentation 2012: Optical, Infrared, and Millimeter Wave, 2012. 84423U, 11 pp. http://dx.doi.org/10. $1117 / 12.926984$.

[23] J.A. Murphy, R. Padman, R. Hills, An experimental submillimetre heterodyne array receiver, Int. J. Infrared Millimeter Waves 9 (4) (1988) 325-350, http:// dx.doi.org/10.1007/BF01013392.

[24] M. Audley et al., Optical measurements of TES bolometers for SAFARI, Proceedings of 23rd International Symposium on Space Terahertz Technology, vol. 2012, 2012, pp. 189-197.

[25] S. Doyle et al., Lumped element kinetic inductance detectors, J. Low Temp. Phys. 151 (1) (2008) 530-536, http://dx.doi.org/10.1007/s10909-007-9685-2.

[26] A. Glindemann, Principles of Stellar Interferometry, Springer, 2011. 